\title{
Research on Intelligent Vehicle Path Tracking with Subsystems Based on Multimodel Intelligent Hierarchical Control Theory
}

\author{
Qing Ye, Ruochen Wang ${ }^{D}$, Chi Zhang, and Yingfeng Cai \\ School of Automobile and Traffic Engineering, Jiangsu University, Zhenjiang 212013, China \\ Correspondence should be addressed to Ruochen Wang; wrc@ujs.edu.cn
}

Received 11 April 2021; Revised 21 May 2021; Accepted 29 May 2021; Published 9 June 2021

Academic Editor: Javier Moreno-Valenzuela

Copyright ( 2021 Qing Ye et al. This is an open access article distributed under the Creative Commons Attribution License, which permits unrestricted use, distribution, and reproduction in any medium, provided the original work is properly cited.

\begin{abstract}
In this paper, a multimodel intelligent hierarchical control (MIHC) algorithm with dual systems is proposed to reduce the performance conflict between a path-tracking motion system and its subsystems during the motion control process of an intelligent vehicle (IV). The working principle of the MIHC algorithm is briefly introduced first, and the dynamic models of IV and the subsystems are constructed. Then, correlation controller models based on MIHC are established. Lastly, the influence of the subsystems on the trajectory tracking of IV is validated through simulations and hardware-in-the-loop test with various condition forms. Results show that the control performance of the automatic steering system has a great influence on the path-tracking accuracy compared with that of the antilock braking system.
\end{abstract}

\section{Introduction}

As an important part of intelligent vehicle (IV) autonomous navigation, IV motion control systems have mainly been investigated in three aspects: lateral motion control $[1,2]$, longitudinal motion control [3], and coordinated motion control [4-6]. Among them, the coordinated motion control system of IV can make the vehicle track the target path with a variable velocity through a certain control strategy, which is of great significance to coordinate the IV path-tracking performance and vehicle-handling stability.

Many studies have presented and elaborated methods for realizing IV path tracking. For example, Huang et al. [7] presented a novel local motion-planning framework in a hierarchical manner for autonomous vehicles to follow a trajectory and avoid obstacles agilely. $\mathrm{Hu}$ et al. [8] investigated the path-tracking control issue for autonomous ground vehicles with integral sliding-mode control (SMC). Tang et al. [9] designed a two-level motion-planning method to schedule the motion of a cable-driven snake robot owning a series-parallel hybrid structure. Hang et al. [10] focused on the path-tracking controller design for autonomous ground vehicles by using four-wheel steering and direct yaw moment control systems, and a linear parameter-varying Ho controller was designed as a high-level controller.

Such studies on IV coordinated motion control systems have been successfully verified in applications, and each applied control strategy can partly improve the vehicle trajectory-tracking accuracy. However, those studies mainly focused on path-tracking motion control, and limited research has been conducted on the coupling mechanism between vehicle kinematics and subsystem dynamics. Some scholars have incorporated the dynamic constraints of vehicle subsystems into the path-tracking control requirements [11-13], but they did not consider the coupling mechanism of the subsystems' dynamic and path-tracking characteristics during the IV movement.

Mutual interference phenomena occur in the lateral and longitudinal motion control of IV during its path-tracking process. In particular, when the vehicle runs at a high velocity under complicated road curvature, the lateral and longitudinal motion control force will be prone to extreme instability. The IV path-tracking system includes the vehicle subsystems, and the subsystems also have nonlinear 
interference. When the vehicle is under steering or braking conditions, the path-tracking accuracy will be greatly affected by the steering accuracy and longitudinal velocity from the automatic steering system (ASS) and antilock braking system (ABS). Consequently, the vehicle might not track the target path in extreme conditions. That is, a strong coupling exists between a path-tracking system and its subsystems. An IV path-tracking control system also includes a motion control system and dynamic subsystems. Existing control strategies, such as adaptive neural network [14], SMC [15], and model predictive control (MPC) $[16,17]$, cannot realize the comprehensive coordination of multiple systems under complex conditions.

In summary, the coupling mechanism between an IV path-tracking system and its subsystems should not be disregarded in the research of IV path-tracking process. Therefore, the innovation of this paper is as follows:

(1) A novel IV path-tracking controller based on multimodel intelligent hierarchical control (MIHC) theory, which considers subsystems, is proposed

(2) The influence of subsystems on the IV path-tracking stability is investigated

To achieve these goals, the working principle of the MIHC algorithm is briefly introduced, and vehicle dynamic models are established in Section 2. In Section 3, an IV trajectory-tracking control algorithm under different hierarchies of MIHC is built. In Section 4, the simulations of the IV trajectory-tracking controller with and without subsystems are compared to verify the influence of subsystems on the accuracy of the IV trajectory-tracking control system. Conclusions are drawn in Section 5.

\section{Path-Tracking Control Framework and Vehicle Model}

2.1. Path-Tracking Control Framework. The framework of the IV trajectory-tracking control system based on MIHC is shown in Figure 1.

The IV trajectory-tracking control system based on MIHC can be divided into three levels: organizational, coordination, and executive. In the organizational level, the backstepping sliding-mode controller outputs the expected generalized control targets by calculating the deviation between the actual vehicle and ideal target state information. The coordination controller determines which subsystem controller works by analyzing the driving condition of the vehicle. The subsystem controller follows the target signal in the executive level.

2.2. Vehicle Dynamic Model. A two degree-of-freedom (DOF) vehicle dynamic model cannot effectively describe the velocity variation of IV, and the IV motion control algorithm based on it cannot meet the movement requirements of all working conditions of IV, such as the velocity variation. Thus, a $3 \mathrm{DOF}$ vehicle dynamic model combined with a nonlinear tire model is established in this study, as shown in Figure 2.

When the lateral acceleration of the vehicle is less than $0.4 \mathrm{~g}$, the $3 \mathrm{DOF}$ vehicle dynamic model is given as follows [18]:

$$
\left\{\begin{array}{l}
\dot{v}_{x}=v_{y} \omega_{c}-\frac{F_{c f} \sin \delta_{f}}{m}, \\
\dot{v}_{y}=\frac{F_{c f} \cos \delta_{f}+F_{c r}}{m}-v_{x} \omega_{c} \\
\dot{\omega}_{c}=\frac{F_{l f} l_{f} \cos \delta_{f}+F_{c r} l_{r}}{I_{Z}}
\end{array}\right.
$$

where $m, v_{x}$, and $v_{y}$ are the mass, longitudinal, and lateral velocities, respectively, $\omega_{c}$ is the yaw velocity, $l_{f}$ and $l_{r}$ are the distances from the center of mass to the front and rear axles, respectively, and $F_{l f}$ and $F_{l r}$ are the longitudinal forces of tires. The tire model is established in accordance with the Dugoff model [19].

2.3. ASS Dynamic Model. The structure of ASS, as the key actuator of the IV path-tracking system, is different, depending on vehicle design and operation requirements. The steering system structure adopted in this study is derived from an electric power steering (EPS) system, in which the steering wheel and associated connections are ignored. The new structure of ASS, including the steering shaft group, electromotor, and gear rack group, is shown in Figure 3.

The dynamic model of ASS can be derived as equation (2) on the basis of traditional EPS dynamic models [20], assuming that the nonlinear factor disturbance in ASS is ignored:

$$
\left\{m_{r} \ddot{x}_{r}+B_{r} \dot{x}_{r}+F_{t r}=\frac{K_{s e}}{r_{p}}\left(\theta_{c}-\frac{x_{r}}{r_{p}}\right), J_{m} \ddot{\theta}_{m}+B_{m} \dot{\theta}_{m}+K_{m}\left(\theta_{m}-g_{m} \theta_{c}\right)=T_{m}\right.
$$

where $\theta_{m}$ and $\theta_{c}$ are the steering angles of the motor and steering spindle, respectively, $B_{r}$ is the damping coefficient of the gear rack group, $K_{m}$ and $K_{s e}$ refer to the stiffness coefficients of the motor and gear rack group, respectively, $m_{r}, J_{m}, r_{p}$, and $F_{t r}$ are the mass of the gear rack group, the moment of inertia of the motor, the radius of the gear, and 


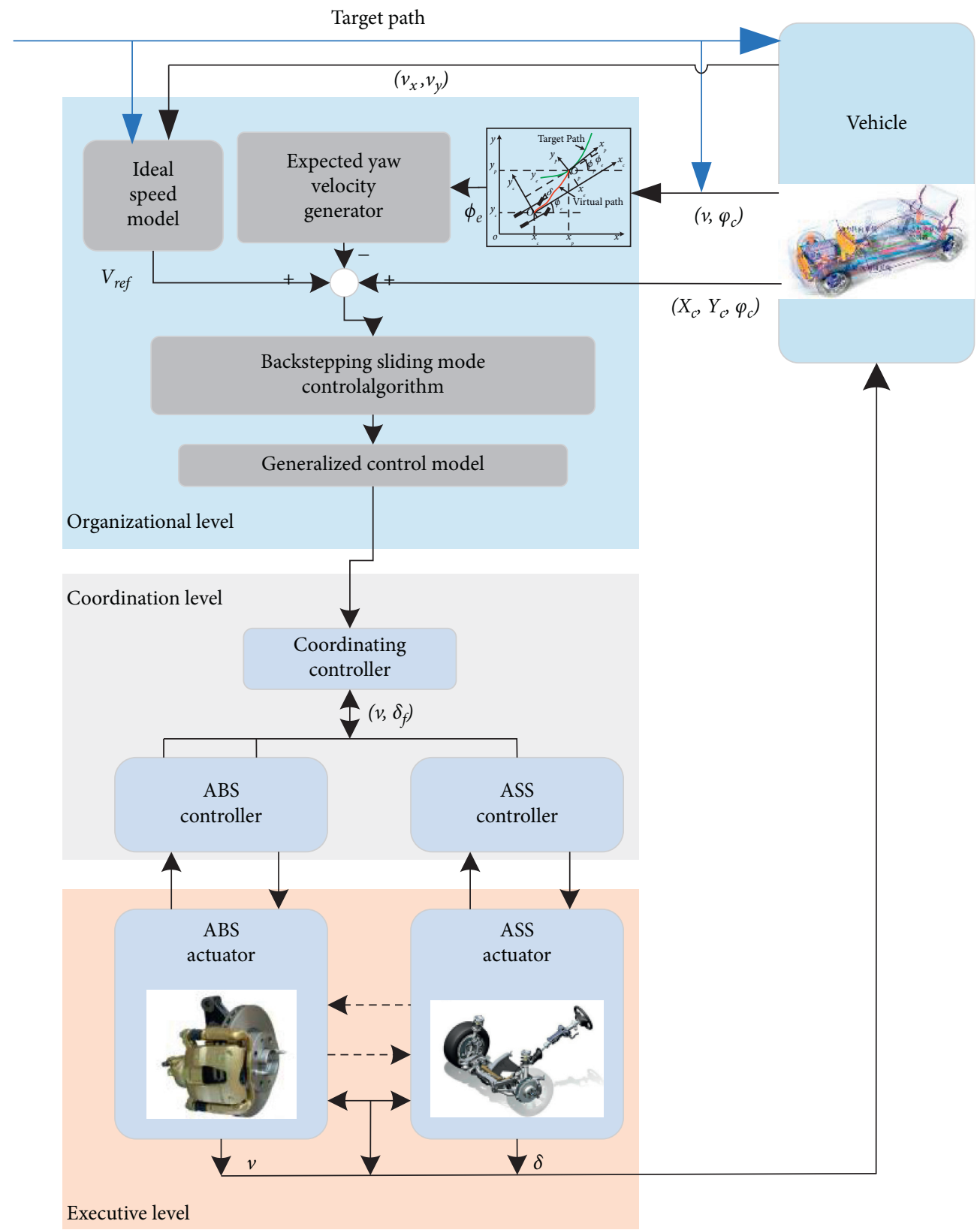

FIgURE 1: Framework of the IV trajectory-tracking control system.

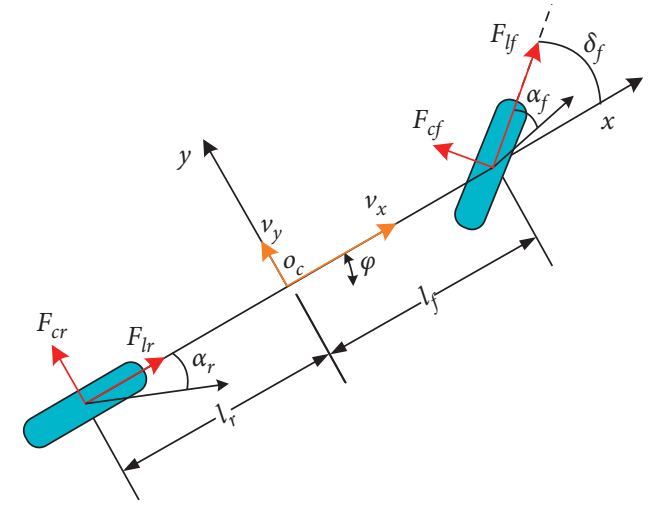

Figure 2: 3DOF vehicle dynamic model. 


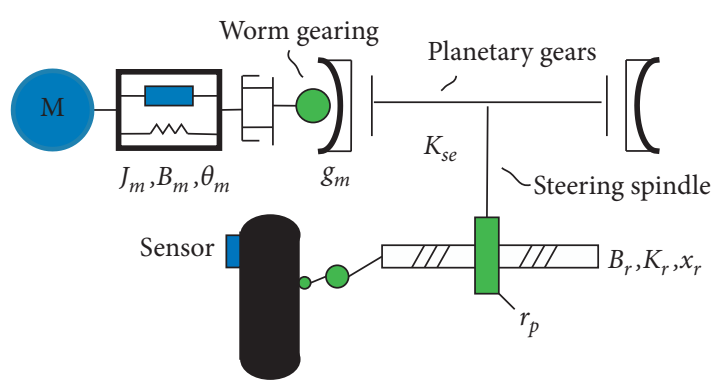

FIgURE 3: ASS dynamic model.

the steering resistance, respectively, $g_{m}$ is the transmission ratio from motion to the steering spindle, and $x_{r}$ is the rack displacement.

$F_{t r}$ and $T_{m}$ can be defined as

$$
\left\{\begin{array}{l}
F_{t r}=K_{r} x_{r} \\
T_{m}=K_{t} I_{m}
\end{array}\right.
$$

where $K_{r}, K_{t}$, and $I_{m}$ refer to the stiffness coefficient of rack, the constant of electromagnetic torque, and the armature current, respectively.

The steering column and motor system are converted into a single deformation of the steering column through mathematical deduction based on the system characteristic in which the torsion characteristics of the steering column and motor system are the same. They can be deduced as

$$
\left\{\begin{array}{l}
K_{s}=\frac{K_{s e} \cdot K_{m}}{g_{m}^{2} K_{m}+K_{s e}} \\
G_{m}=\frac{r_{p}}{g_{m}} .
\end{array}\right.
$$

Then, equation (2) can be derived as

$$
\left\{m_{r} \ddot{x}_{r}+B_{r} \dot{x}_{r}+K_{r} x_{r}=\frac{K_{s}}{G_{m}}\left(\theta_{m}-\frac{x_{r}}{G_{m}}\right), J_{m} \ddot{\theta}_{m}+B_{m} \dot{\theta}_{m}+K_{s}\left(\theta_{m}-\frac{x_{r}}{G_{m}}\right)=-T_{m},\right.
$$

where $K_{s}$ and $G_{m}$ refer to the comprehensive torsional deformation stiffness and transmission ratio, respectively. The PD control strategy is considered the ABS control strategy based on its simple structure, good stability, and convenient adjustment. The armature current $I_{m}$ can be described as

$$
I_{m}=K_{p}\left(\delta-\delta_{f}\right)+K_{D}\left(\dot{\delta}-\dot{\delta}_{f}\right) .
$$

In equation (6), the tire steering angle is linearly correlated with the rack displacement:

$$
\left\{\begin{array}{l}
\delta=x_{r} \cdot i_{0}, \\
i_{0}=\frac{1}{L}
\end{array}\right.
$$

2.4. ABS Dynamic Models. The tire dynamic model is shown in Figure 4.

Ignoring the tire rolling and air resistance, the ABS dynamic models are shown as follows [21]:

$$
\left\{\begin{array}{l}
I_{w} \dot{\omega}_{w}=R F_{x}-T_{b}, \\
m \dot{V}_{\mathrm{car}}=-F_{x}, \\
F_{x}=\mu F_{z},
\end{array}\right.
$$

where $I_{w}, R, \mu$, and $\omega_{w}$ are the tire inertia, radius, adhesion coefficient, and angular velocity, respectively and $F_{z}, T_{b}$, and $F_{x}$ are the vertical load, braking torque, and longitudinal friction of the tire, respectively. A nonlinear relationship exists between the road adhesion coefficient and the tire slip rate on the basis of the Dugoff dynamic model. The linear curve of the road adhesion coefficient and tire slip rate is shown in Figure 5.

$$
\begin{cases}\mu=\frac{\mu_{h}}{\mu_{g}} S, & S \leq S_{0}, \\ \mu=\frac{\mu_{h}-\mu_{g} S_{0}}{1-S_{0}}-\frac{\mu_{h}-\mu_{g}}{1-S_{0}} S, & S>S_{0}\end{cases}
$$

where $S_{0}$ refers to the vehicle slip rate corresponding to the peak value of the longitudinal adhesion coefficient and $\mu_{h}$ and $\mu_{g}$ are the tire peak longitudinal adhesion coefficient and the tire longitudinal adhesion coefficient when the wheel is fully locked (the slip rate is 1), respectively.

In the actual vehicle braking process, the ground braking torque is determined by the ABS torque but restricted by the ground adhesion coefficient. When the braking torque is greater than the maximum ground braking torque, the wheel appears to be locked and dragged, which results in actual inequality of the vehicle and wheel velocities [21]. The vehicle longitudinal slip rate can be expressed as

$$
S=\frac{V_{\mathrm{car}}-V_{w}}{V_{\mathrm{car}}}
$$

where $V_{\text {car }}$ and $V_{w}$ are the vehicle and wheel velocities, respectively, with

$$
V_{w}=\omega_{w} \cdot R
$$

Figure 5 depicts a linear correlation of the change rate between the tire adhesion coefficient and slip rate under local conditions, which can be obtained as 


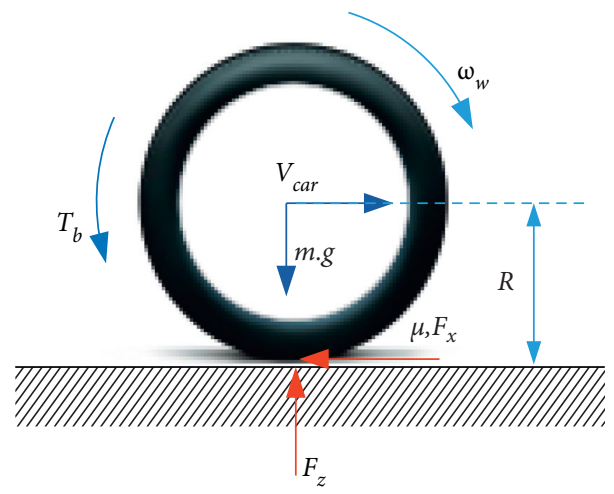

Figure 4: Tire model.

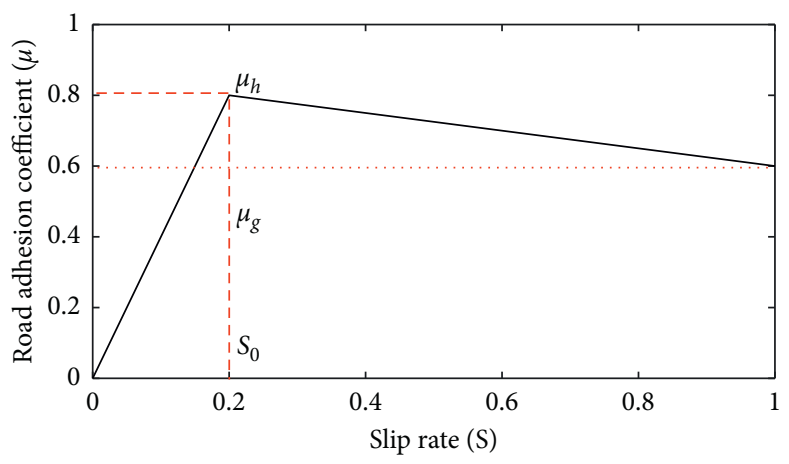

Figure 5: The linear curve of the road adhesion coefficient and the tire slip rate.

$$
\dot{\mu}=k_{\mu-S} \dot{S}
$$

where $k_{\mu-S}$ refers to the correlation coefficient. $k_{\mu-S}$ can be positive or negative under different cases on the basis of the relationship between the tire adhesion coefficient and wheel slip rate.

The change rate of the tire longitudinal adhesion coefficient $\dot{\mu}$ can be then obtained from equations (11) and (12):

$$
\dot{\mu}=-k_{\mu-S} \frac{R}{V} \dot{\omega}_{w} .
$$

In accordance with the working principle of ABS, the braking system controls the system slip rate by adjusting the solenoid valve in real time to keep the system slip rate near the optimal slip rate. The change rate of brake torque is proportional to the fluid flow rate, and the flow rate is proportional to the control valve opening. Therefore, the brake pressure rate is proportional to the valve command, and the change rate of brake torque can be expressed as follows:

$$
\dot{T}_{b}=U_{w} .
$$

Then, the ABS controller is designed on the basis of the above research. The PD control strategy is considered, in which the specific expression is as follows:

$$
U_{w}=K_{p w}\left(\omega_{w}-\omega_{\text {wdes }}\right)+K_{d w}\left(\dot{\omega}_{w}-\dot{\omega}_{\text {wdes }}\right),
$$

where $\omega_{\text {wdes }}$ refers to the desired wheel velocity. The dynamic model of ABS can be derived as

$$
\ddot{\omega}_{w}+\frac{k_{\mu-S} R^{2} F_{z}}{V I_{w}} \dot{\omega}_{w}+\frac{K_{p w}}{I_{w}}\left(\omega_{w}-\omega_{\text {wdes }}\right)+\frac{K_{d w}}{I_{w}}\left(\dot{\omega}_{w}-\dot{\omega}_{\text {wdes }}\right)=0 .
$$

\section{Path-Tracking Control System}

In accordance with the working principle of the MIHC system, different controllers under various levels are shown in this section. They include the backstepping sliding-mode controller (organizational level) and coordination controller.

\subsection{Organizational Level}

3.1.1. Lateral Motion Reference Model. The path-tracking algorithm is the key part of IV motion control. Scholars have studied it with different methods, such as adaptive neural network [13], SMC [14], and MPC [15]. Existing literature on path-tracking algorithms has mostly adopted the visual preview model, which has a limitation, i.e., the tracking accuracy of the vehicle motion system is greatly affected by preview distance and road curves. To reduce the influence of external interference on the path-tracking accuracy, a pathtracking algorithm based on the expected yaw velocity is adopted in this study. This path-tracking algorithm assumes that a virtual path exists between vehicle and preview point locations, as shown in Figure 6. 
The position and orientation errors between vehicle and preview point locations under the road coordinate system can be derived as equation (17) on the basis of geometric theory [15]:

$$
p=\left[\begin{array}{l}
x_{e} \\
y_{e} \\
\phi_{e}
\end{array}\right]=\left[\begin{array}{ccc}
\cos \phi_{c} & \sin \phi_{c} & 0 \\
-\sin \phi_{c} & \cos \phi_{c} & 0 \\
0 & 0 & 1
\end{array}\right]\left[\begin{array}{l}
x_{p}-x_{c} \\
y_{p}-y_{c} \\
\phi_{p}-\phi_{c}
\end{array}\right],
$$

where $y_{e}$ and $x_{e}$ refer to the lateral deviation and preview distance, respectively, $\varphi_{c}$ and $\varphi_{p}$ are the vehicle yaw angles at the vehicle and preview points, respectively, $\varphi_{e}$ is the orientation deviation between $\varphi_{c}$ and $\varphi_{p}$, and $y_{e}$ and $x_{e}$ in the vehicle coordinate system can be deduced as follows [15]:

$$
\left\{\begin{array}{l}
\dot{y}_{e}=-v_{y}+x_{e} \varepsilon-x_{e} \omega_{c}, \\
\dot{\varepsilon}=v_{x} \rho-\omega_{c}
\end{array}\right.
$$

where $\rho$ and $\varepsilon$ refer to the road curvature and orientation deviation, respectively.

Many possibilities may occur for the virtual path between vehicle and preview point locations under the vehicle coordinate system. The vehicle-handling performance and path-tracking efficiency are considered in this study to restrict the curve shape of the virtual path. That is, the virtual curve is symmetric, and the vehicle enters or leaves the virtual path smoothly. The constraint conditions of the virtual path are defined as follows:

$$
\left\{\begin{array}{l}
y(0)=0, \\
\dot{y}(0)=0, \\
y\left(x_{e}\right)=y_{e},\left.\right|_{x=0}=\frac{\omega_{c}}{v}, y\left(\frac{x_{e}}{2}\right)=\frac{y_{e}}{2}, v=\sqrt{v_{x}^{2}+v_{y}^{2}}, \\
\frac{\ddot{y}}{\left(1+\dot{y}^{2}\right)^{1.5}} .
\end{array}\right.
$$

The quadratic polynomial curvilinear of the virtual driving path can be obtained as

$$
y(x)=\frac{\omega_{c} \cdot x_{e}^{2}-6 \cdot v \cdot y_{e}}{v \cdot x_{e}^{4}} x^{4}+\frac{-3 \cdot \omega_{c} \cdot x_{e}^{2}+14 \cdot v \cdot y_{e}}{2 \cdot v \cdot x_{e}^{3}} x^{3}+\frac{\omega_{c}}{2 v} x^{2} .
$$

3.1.2. Longitudinal Motion Reference Model. The tracking accuracy of the vehicle motion system is greatly affected not only by the control algorithm but also by the vehicle velocity. Particularly, when the vehicle runs at a high velocity under complicated road curvature, the tire grounding property will deteriorate, and vehicle rollover easily occurs in extreme cases. Hence, this study provides the expected velocity model in equation (21) based on the human driver behavior simulation algorithm [22] to prevent the above situation:

$$
a_{\mathrm{ref}}=\frac{v_{\mathrm{ref}}^{2}-v_{x}^{2}}{2 L}
$$

where $a_{\text {ref }}$ and $v_{\text {ref }}$ refer to the reference longitudinal acceleration and velocity, respectively. $v_{\text {ref }}$ can be defined as follows [22]:

$$
v_{\text {ref }}=v_{0}-v_{0} e^{-\zeta R}
$$

where $v_{0}$ and $\zeta$ are the initial braking velocity and the experience factor, respectively.

3.1.3. Backstepping Sliding-Mode Controller. Assuming that the IV tracks the designed virtual path without deviation, the ideal yaw velocity can be derived as

$$
\omega_{d}=v \cdot \rho_{v r}
$$

where $\rho_{v r}$ is the road curvature, and the change rate of road curvature in the virtual path based on the calculation formula of road curvature can be shown as follows:

$$
\begin{aligned}
\dot{\rho}_{v r}= & \frac{24 a_{1} x+6 a_{2}}{A(x)^{(3 / 2)}} \\
& -\frac{12\left(6 a_{1} x^{2}+3 a_{2} x+a_{3}\right)^{2}\left(4 a_{1} x^{3}+3 a_{2} x^{2}+2 a_{3} x\right)}{A(x)^{(5 / 2)}},
\end{aligned}
$$

with

$$
\begin{aligned}
A(x)= & 16 a_{1}^{2} x^{6}+24 a_{1} a_{2} x^{5}+16 a_{1} a_{3} x_{4}+9 a_{2}^{2} x^{4} \\
& +12 a_{2} a_{3} x^{3}+4 a_{3}^{2} x^{2}+1 .
\end{aligned}
$$

The change rate of ideal yaw velocity can be given as

$$
\dot{\omega}_{d}=\dot{v} \cdot \rho_{v r}+v \cdot \dot{\rho}_{v r}=\frac{\dot{v} \omega_{c}}{v}+v\left\{\begin{array}{c}
\frac{24 a_{1} x_{e}+6 a_{2}}{A\left(x_{e}\right)^{(3 / 2)}} \\
-\frac{12\left(6 a_{1} x_{e}^{2}+3 a_{2} x_{3}+a_{3}\right)^{2}\left(4 a_{1} x_{e}^{3}+3 a_{2} x_{e}^{2}+2 a_{3} x_{e}\right)}{A\left(x_{e}\right)^{(5 / 2)}}
\end{array}\right\} .
$$




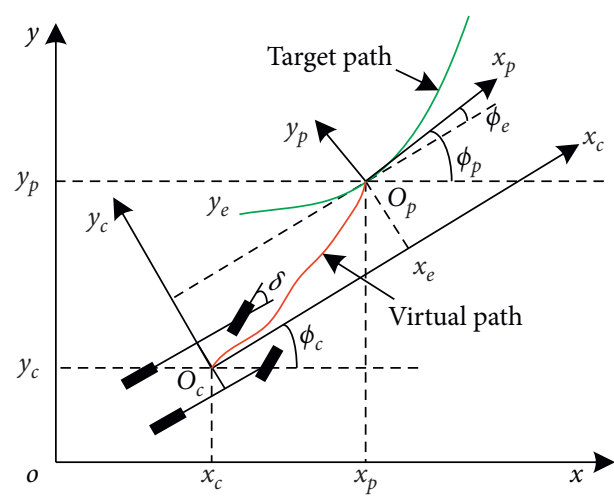

FIGURE 6: Vehicle path model.

Consequently, the expected yaw velocity can be expressed as

$$
\omega_{r}=\omega_{c}+\lambda \dot{\omega}_{d}
$$

where $\lambda$ is the coefficient of proportionality, which is related to control interval time.
Lastly, the organizational level controller is designed, which is named longitudinal and later motion cooperative controller. The SMC algorithm is considered in this section to track the expected yaw velocity and the expected velocity. However, the expected yaw velocity model, which is presented in equation (27), is of higher order and nonlinear degree, and the traditional SMC algorithm could not realize effective tracking control. To solve this problem, backstepping theory is adopted on the basis of its effect on the reduction in the order of the higher-order mathematical model [23]. The backstepping SMC algorithm is then proposed here to design the desired yaw velocity-tracking controller.

The sliding-mode switch function is defined as

$$
\left\{\begin{array}{l}
z_{1}=v_{x}-v_{\text {ref }} \\
z_{2}=\omega_{c}-\omega_{r}
\end{array}\right.
$$

The derivative of equation (28) can be derived from equations (22) and (27) in accordance with calculus theory:

$$
\left\{\begin{array}{l}
\dot{z}_{1}=\dot{v}_{x}-\dot{v}_{\text {ref }}=\frac{v_{\text {ref }}^{2}-v_{x}^{2}}{2 L}, \\
\dot{z}_{2}=\dot{\omega}_{c}-\dot{\omega}_{r}=\lambda \dot{\omega}_{c}\left(\frac{120}{x_{e} v A^{1.5}}+\frac{36 B_{11} B_{12}}{x_{e}^{5} v^{3} A^{2.5}}+\frac{48 B_{21}^{2}}{x_{e}^{3} v^{3} A^{2.5}}+\frac{120 B_{21}^{2} B_{22} B_{12}}{x_{e}^{7} v^{5} A^{3.5}}-\frac{288 B_{21} B_{22}}{x_{e}^{7} v^{5} A^{3.5}}\right),
\end{array}\right.
$$

with

$$
\left\{\begin{array}{l}
A=\frac{\omega_{c}^{2} x_{e}^{4}-12 v \omega_{c} x_{e}^{2} y_{e}+4 v^{2} x_{e}^{2}+36 v^{2} y_{e}^{2}}{v^{2} x_{e}^{2}} \\
B_{11}=-5 \omega_{c} x_{e}^{2}+34 v y_{e}, \\
B_{12}=2 \omega_{c} x_{e}^{4}-12 v x_{e}^{2} y_{e}, \\
B_{21}=2 v x_{e}^{2}+3 \omega_{c} x_{e}^{2}-30 v y_{e}, \\
B_{22}=-\omega_{c} x_{e}^{2}+6 v y_{2} .
\end{array}\right.
$$

The stability parameter is defined as

$$
\left\{\begin{array}{l}
\alpha_{1}=c_{1} z_{1}, \\
\alpha_{2}=c_{2} z_{2},
\end{array}\right.
$$

where $c_{1}$ and $c_{2}$ are the positive constants, and the Lyapunov function of equation (28) is given as

$$
\left\{\begin{array}{l}
V_{1}=\frac{1}{2} z_{1}^{2}, \\
V_{2}=\frac{1}{2} z_{2}^{2} .
\end{array}\right.
$$
as

The derivative function of equation (32) can be obtained

$$
\left\{\begin{array}{l}
\dot{V}_{1}=z_{1} \dot{z}_{1}=z_{1}\left(z_{1}^{\prime}-\alpha_{1}\right)=z_{1} z_{1}^{\prime}-c_{1} z_{1}^{2} \\
\dot{V}_{2}=z_{2} \dot{z}_{2}=z_{2}\left(z_{2}^{\prime}-\alpha_{2}\right)=z_{2} z_{2}^{\prime}-c_{2} z_{2}^{2}
\end{array}\right.
$$

with

$$
\left\{\dot{z}_{1}^{\prime}=\ddot{z}_{1}+\dot{\alpha}_{1}, \dot{z}_{2}^{\prime}=\ddot{z}_{2}+\dot{\alpha}_{2}\right. \text {. }
$$

The Lyapunov function of equation (32) is given as

$$
\left\{\begin{array}{l}
V_{1}^{\prime}=V_{1}+\frac{1}{2} \sigma_{1}^{2}, \\
V_{2}^{\prime}=V_{2}+\frac{1}{2} \sigma_{2}^{2},
\end{array}\right.
$$

where $\sigma_{1}$ and $\sigma_{2}$ are the switching function, which can be expressed as

$$
\left\{\begin{array}{l}
\sigma_{1}=k_{1} z_{1}+z_{1}^{\prime} \\
\sigma_{2}=k_{2} z_{2}+z_{2}^{\prime}
\end{array}\right.
$$

In equation (37), $k_{1}$ and $k_{2}$ are always greater than zero on the basis of calculation, which means that the derivative function of $V_{1}^{\prime}$ and $V_{2}^{\prime}$ can be obtained as 


$$
\left\{\begin{array}{l}
\dot{V}^{\prime}{ }_{1}=\dot{V}_{1}+\sigma_{1} \dot{\sigma}_{1}=z_{1} z_{1}^{\prime}-c_{1} z_{1}^{2}+\sigma_{1}\left(k_{1} \dot{z}_{1}+\dot{z}^{\prime}{ }_{1}\right), \\
\dot{V}^{\prime}{ }_{2}=\dot{V}_{2}+\sigma_{2} \dot{\sigma}_{2}=z_{2} z_{2}^{\prime}-c_{2} z_{2}^{2}+\sigma_{2}\left(k_{2} \dot{z}_{2}+\dot{z}^{\prime}{ }_{2}\right) .
\end{array}\right.
$$

The target function of the IV longitudinal and lateral motion system can be derived as

$$
\left[\begin{array}{c}
f\left(v_{x}\right) \\
f\left(\omega_{c}\right)
\end{array}\right]=\left[\begin{array}{c}
f\left[\left(v_{x}-v_{\mathrm{ref}}\right)\left(k_{1}+c_{1}\right)+\dot{v}_{x}-\dot{v}_{\mathrm{ref}}=0\right] \\
f\left[\left(\omega_{c}-\omega_{r}\right)\left(k_{1}+c_{1}\right)+\dot{\omega}_{c}-\dot{\omega}_{r}=0\right]
\end{array}\right] .
$$

3.2. Coordination Level. During the actual driving process of IVs, the subsystems will work or not under different road conditions. For example, when a vehicle is running at a straight line with constant velocity, ABS and ASS will not work. When the vehicle is running under braking or understeering conditions, ABS will work to ensure the tracking accuracy of the vehicle velocity. When the vehicle is running in an oversteering condition, ABS and ASS will work simultaneously to improve the vehicle-handling stability and path-tracking performance. The theoretical values of front wheel angle $\left(\delta_{f}\right)$ and vehicle acceleration $\left(a_{\text {ref } L}\right)$ are the evaluation criteria for the design of a coordination controller, as shown in Table 1.

In Table $1, \delta_{f L}$ and $a_{\text {ref } L}$ are the positive numbers infinitely close to 0 , and the steering stability factor $K$ can be given as

$$
K=\frac{m}{\left(l_{f}+l_{r}\right)^{2}}\left(\frac{l_{r}}{C_{y r}}-\frac{l_{f}}{C_{y f}}\right),
$$

where $C_{y f}$ and $C_{y r}$ refer to the lateral stiffness of the front and rear wheels, respectively.

\section{Simulation Analysis}

In this section, the single lane change (SLC) simulations of the IV path-tracking system are implemented on the MATLAB/Simulation platform. The road curvature of SLC is shown in Figure 7. Different road and driving conditions are adopted in the simulation implementation. The research objective is to analyze the influence of subsystems on the IV path-tracking stability. First, the comparative simulation of the IV path-tracking system with or without subsystems under the same velocity condition is performed. Second, the influence of each subsystem on the tracking performance and stability of the IV path-tracking system under different vehicle velocity conditions is studied. Lastly, the external interference torque is evaluated to investigate the influence of the subsystems on the stability of the IV path-tracking system. The controller parameters are set under an independent condition, which means the control parameters of the subsystems set in Section 2 do not consider the impact of path-tracking motion. The values of the ASS control parameters $K_{p}$ and $K_{d}$ are set to 18.4 and 11.1, respectively. The values of $K_{p w}$ and $K_{d w}$ in ABS are 1163.7 and 48.2, respectively, by debugging the parameters of the controller.
The corresponding parameters of the vehicle and road are listed in Table 2.

4.1. With or without Subsystems. The vehicle velocity in the SLC simulation is shown in Figure 8(c), with the simulation condition that IV is tracking the target path with or without subsystems. The lateral deviation, orientation deviation, and velocity results are plotted in Figures 8(a) and 8(b). A1, A2, $\mathrm{A} 3$, and $\mathrm{A} 4$ refer to the IV path-tracking system without subsystems, with ASS, with ABS, and with both subsystems, respectively.

Figure 8 shows the transient response of lateral and orientation deviations of $\mathrm{A} 1, \mathrm{~A} 2, \mathrm{~A} 3$, and $\mathrm{A} 4$ for the reference trajectory at $72 \mathrm{~km} / \mathrm{h}$. The steady-state lateral deviation of A1 is within $\pm 0.25 \mathrm{~m}$, and the absolute peak value is $0.23 \mathrm{~m}$. The peak value of lateral deviation in A3 increases to $0.231 \mathrm{~m}$, which indicates that the small velocity changes caused by ABS have almost no influence on the accuracy of the IV path-tracking system. The peak lateral deviations of $\mathrm{A} 1$ and $\mathrm{A} 2$ are 0.23 and $0.326 \mathrm{~m}$, respectively. These values imply that the accuracy of the IV path-tracking system is greatly influenced by that of ASS under the same velocity conditions compared with that of ABS. The vehicle velocity is variable, which makes the curves of $\mathrm{A} 2$ and $\mathrm{A} 4$ show fluctuations and be stable rapidly. From A2 and A4, the new controller can amplify the fluctuation results of ASS.

4.2. SLC Motion with Different Velocities. The velocity conditions are considered to analyze the influence of velocity on the IV trajectory-tracking accuracy quantitatively. The different subsystems are also considered under diverse velocities. The analysis includes the comparative simulation of the IV path-tracking system with or without ASS, with or without ABS, and with or without both subsystems. The values of the control parameters are the same as those in Section 4.1.

4.2.1. Comparative Simulation of the IV Path-Tracking System with or without ASS. The influence of ASS on the stability and accuracy of the IV path-tracking system under different velocity conditions is considered. This simulation assumes that the vehicle completes an SLC maneuver at the fixed velocities of 18,36 , and $72 \mathrm{~km} / \mathrm{h}$. The comparison of lateral and orientation deviations is shown in Figure 9. In this Figure, S1 and S2 refer to the IV path-tracking system with and without ASS, respectively.

Figure 9 demonstrates that the peak absolute lateral deviations of S1 and S2 at $18 \mathrm{~km} / \mathrm{h}$ are 0.046 and $0.047 \mathrm{~m}$, respectively. The corresponding data at 36 and $72 \mathrm{~km} / \mathrm{h}$ are $0.092,0.095,0.19$, and $0.201 \mathrm{~m}$, which mean that ASS greatly influences the accuracy of the IV path-tracking system as the velocity increases.

4.2.2. Comparative Simulation of the IV Path-Tracking System with or without ABS. This simulation assumes that the vehicle completes an SLC maneuver at varying velocity conditions (Figure 10(c)). A comparison of lateral and 
TABLE 1: Evaluation criteria for the design of a coordination controller.

\begin{tabular}{|c|c|c|c|c|}
\hline Road condition & \multicolumn{2}{|c|}{ Driving condition } & Driving mode & Subsystem \\
\hline \multirow[b]{2}{*}{ Straight condition } & $\left|\delta_{f}\right| \leq \delta_{f L}$ & Normally & 1 & - \\
\hline & $\begin{aligned} a_{\mathrm{ref}} & \leq a_{\mathrm{ref} L} \\
\left|\delta_{f}\right| & \leq \delta_{f L}, \\
a_{\mathrm{ref}} & \leq a_{\mathrm{ref} L}\end{aligned}$ & Brake & 2 & ABS \\
\hline \multirow[t]{2}{*}{ Steering condition } & $\begin{array}{c}\left|\delta_{f}\right|>\delta_{f L} \\
K>0\end{array}$ & Understeer & 3 & ASS \\
\hline & $\begin{array}{c}\left|\delta_{f}\right|>\delta_{f L} \\
K>0\end{array}$ & Oversteer & 4 & $\mathrm{ABS}+\mathrm{ASS}$ \\
\hline
\end{tabular}

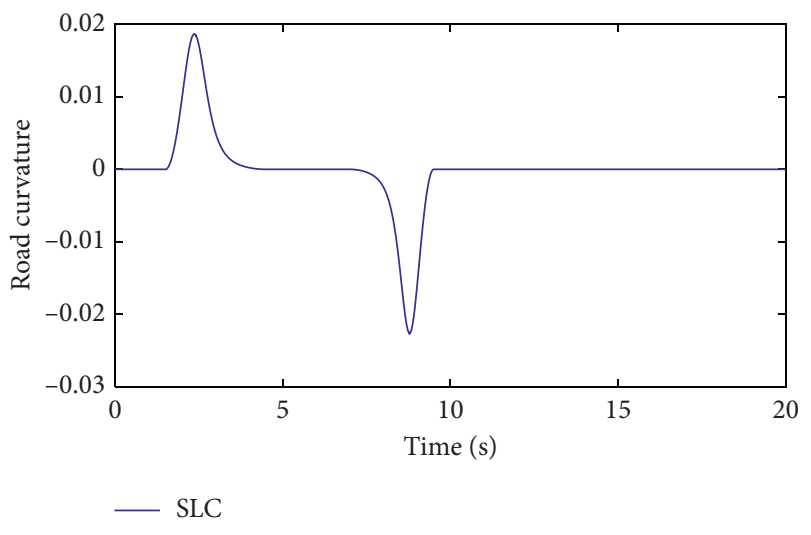

Figure 7: Road curvature of SLC.

orientation deviations is shown in Figure 10. In this figure, $B 1$ refers to the IV path-tracking system without ABS under a fixed velocity; B2 and B3 refer to that with and without ABS under varying velocity conditions ( $36 \mathrm{~km}$ tops), respectively.

Figure 10 (c) presents that the target velocity change of IV is relatively small on the basis of equation (22). The actual velocity change of IV is smaller than that of the target due to the inherent characteristics of ABS. The simulation result shows that the errors of lateral and orientation deviations between $\mathrm{B} 1$ and $\mathrm{B} 3$ are minimal when the ABS parameters are fixed. That is, ABS has a small influence on the accuracy of the IV path-tracking system.

However, the vehicle target velocity may change rapidly and dramatically in accordance with the virtual driver's demand under special working conditions, such as obstacle avoidance. In this section, rapid velocity change conditions are considered to study the influence of ABS on the accuracy and robustness of the IV pathtracking system. This simulation assumes that the vehicle runs at a speed of $72 \mathrm{~km} / \mathrm{h}$ for $3 \mathrm{~s}$ and then continues to run at a speed of $36 \mathrm{~km} / \mathrm{h}$, as shown in Figure 11(c). A comparison of lateral and orientation deviations is shown in Figure 11. In this figure, $\mathrm{C} 1$ refers to the IV pathtracking system without ABS under a fixed velocity; C2 and $\mathrm{C} 3$ refer to that with and without $\mathrm{ABS}$ under the given velocity condition, respectively.

Figure 11 indicates that the curves of C1, C2, and C3 are almost the same in the first $3 \mathrm{~s}$ of simulation. The absolute peak values of lateral and orientation deviations of $\mathrm{C} 1$ are higher than those of $\mathrm{C} 2$ and $\mathrm{C} 3$. The results are consistent with those in Figure 9. The absolute peak values of orientation and lateral deviations in $\mathrm{C} 2$ are $0.11 \mathrm{rad} / \mathrm{s}$ and $0.09 \mathrm{~m}$, respectively, during the last $7 \mathrm{~s}$. The relevant values of $\mathrm{C} 3$ are $0.13 \mathrm{rad} / \mathrm{s}$ and $0.106 \mathrm{~m}$. These results imply that $\mathrm{ABS}$ has a certain influence on the accuracy of the IV path-tracking system when the velocity changes dramatically. The velocity-tracking performance is relatively poor in accordance with the inherent characteristics of ABS. The curve fluctuation of $\mathrm{C} 2$ is more obvious than that of $\mathrm{C} 3$ when the velocity changes dramatically, especially in the third simulation second, which indicates that ABS can improve the robustness performance of the IV path-tracking system.

\subsubsection{Comparative Simulation of the IV Path-Tracking} System with or without Both Subsystems. Lastly, a comparative simulation of the IV path-tracking system with or without both subsystems is considered, as shown in Figure 12. This simulation assumes that the vehicle completes an SLC maneuver at varying velocity conditions. The initial velocities of the vehicle are set to 36 and $72 \mathrm{~km} / \mathrm{h}$. In the figure, V1 and V2 refer to the IV path-tracking system with and without subsystems, respectively.

Figure 12 shows a certain difference between V1 and V2 under the same speed condition. When the vehicle velocity is $36 \mathrm{~km} / \mathrm{h}$, the peak deviation values of $\mathrm{V} 1$ and $\mathrm{V} 2$ are 0.098 and $0.106 \mathrm{~m}$, respectively. The corresponding values at $72 \mathrm{~km} / \mathrm{h}$ are 0.23 and $0.33 \mathrm{~m}$. The curve of V2 shows an evident fluctuation when the velocity is $72 \mathrm{~km} / \mathrm{h}$. The simulation results are consistent with the analysis results in Figures 8 and 9. In sum, ASS has a great influence on the accuracy of the IV path-tracking system as the velocity increases, and the new controller can amplify the fluctuation results of ASS.

4.3. SLCMotion with Different Interference Torques. After the influence of the subsystems on the accuracy and stability of the IV path-tracking system is analyzed under different velocity conditions, the torque interference caused by ASS is also considered. This simulation assumes that the vehicle runs at varying speeds $(36 \mathrm{~km} / \mathrm{h}$ tops), and the interference torque is set to 0,40 , and $50 \mathrm{~N}$. The results are illustrated in Figure 13.

Figure 13 presents that when the vehicle turns steadily, the curves under different interference torques are almost the same. This result means that the interference torque has a minimal influence on the stability and accuracy of the IV path-tracking 
Table 2: Parameters.

\begin{tabular}{lcc}
\hline Parameters & Units & Values \\
\hline Vehicle mass $(\mathrm{m})$ & $\mathrm{kg}$ & 1963 \\
Yaw inertia $\left(I_{z}\right)$ & $\mathrm{kg} \cdot \mathrm{m}^{2}$ & 3048 \\
Scale factors $\left(c_{1}, \lambda, \eta\right.$, and $\left.k_{1}\right)$ & - & $5 ; 0.01 ; 15 ; 20$ \\
Distance $1\left(l_{f}\right.$ and $\left.l_{r}\right)$ & $\mathrm{m}$ & $1.232 ; 1.468$ \\
Distance $2\left(t_{f}\right.$ and $\left.t_{r}\right)$ & $\mathrm{m}$ & $1.535 ; 1.535$ \\
Distance $3\left(L\right.$ and $\left.r_{p}\right)$ & $\mathrm{m}$ & $0.24 ; 0.0078$ \\
Height $(h)$ & $\mathrm{m}$ & 0.505 \\
Gear rack mass $\left(m_{r}\right)$ & $\mathrm{kg}$ & 2.5 \\
Moment of inertia $\left(J_{m}\right)$ & $\mathrm{N} \cdot \mathrm{m} \cdot \mathrm{s} / \mathrm{rad}$ & 0.0016 \\
Damping coefficient $\left(B_{m}\right.$ and $\left.B_{r}\right)$ & $\mathrm{N} \cdot \mathrm{m} / \mathrm{s} / \mathrm{rad}$ & $0.034 ; 312$ \\
Stiffness coefficient $\left(K_{s e}, K_{m}\right.$, and $\left.K_{r}\right)$ & - & $\mathrm{V} \cdot \mathrm{s} / \mathrm{rad}$ \\
Combined transmission ratio $\left(g_{m}\right)$ & $\mathrm{N} \cdot \mathrm{s} / \mathrm{A}$ & $123 ; 125 ; 29871$ \\
Back EMF constant $\left(K_{e}\right)$ & $\Omega$ & 16.5 \\
Electromagnetic torque constant $\left(K_{t}\right)$ & - & 0.0345 \\
Inductance of armature winding $\left(L_{m}\right)$ & 0.0345 \\
Experience factor $(\zeta)$ & 0.035
\end{tabular}

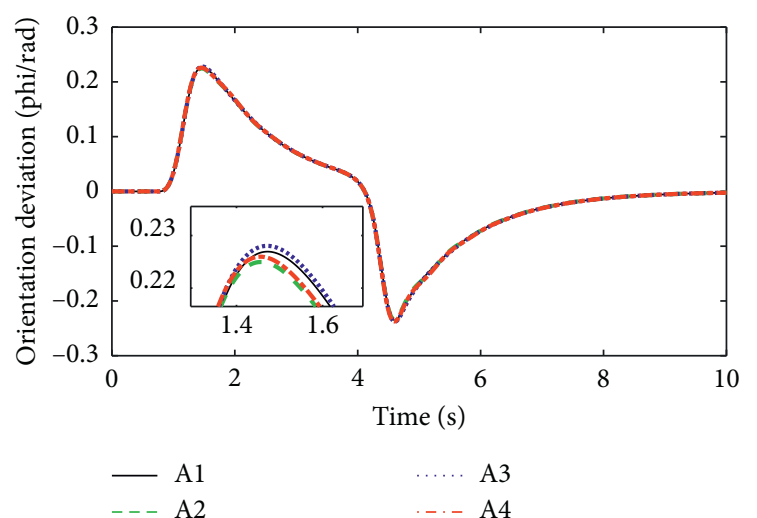

(a)
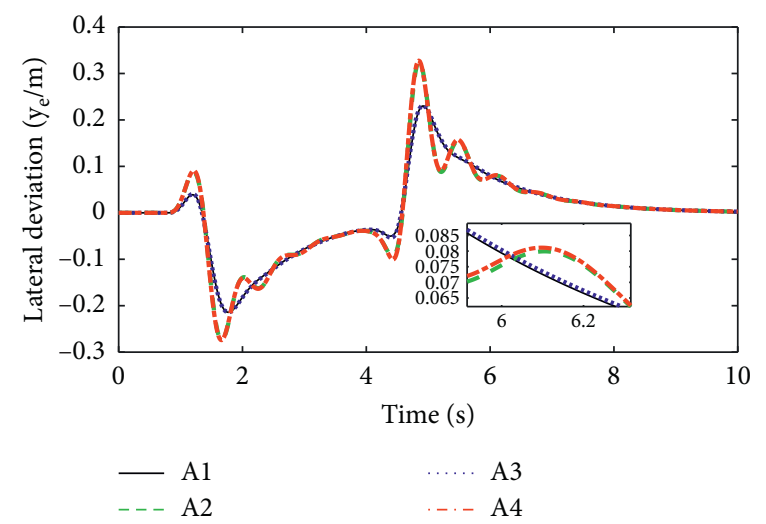

(b)

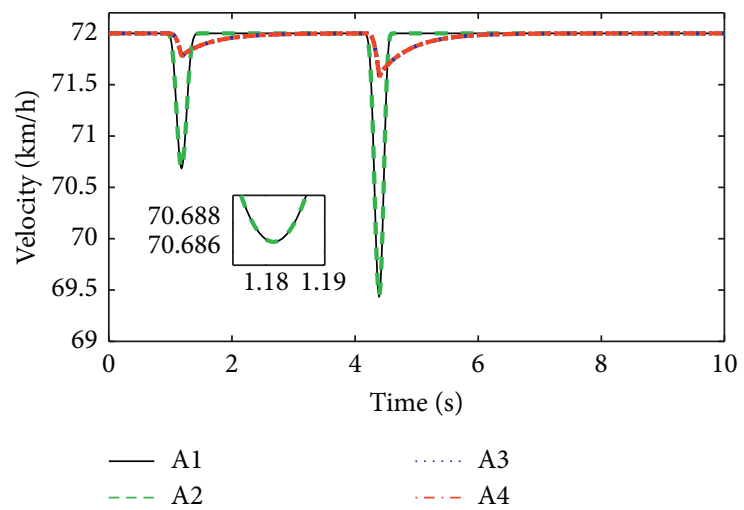

(c)

Figure 8: The effect comparison between A1, A2, A3, and A4. (a) Orientation deviation. (b) Lateral deviation. (c) Velocity.

system. When the vehicle changes the steering direction of tires, the direction of interference torque on tires is changed, which can make the instantaneous tracking performance of IV jitter evidently. The absolute values of lateral deviation are $0.1,0.188$, and $0.215 \mathrm{~m}$ in the course of steering mutation. The curves in Figure 13 converge fast on the basis of the design controller, which indicates the superior control performance of the IV path-tracking system. 


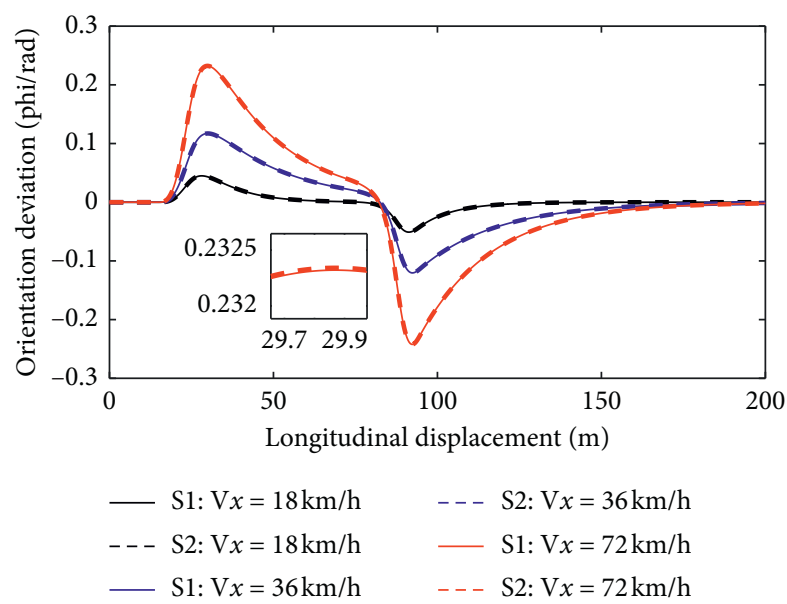

(a)

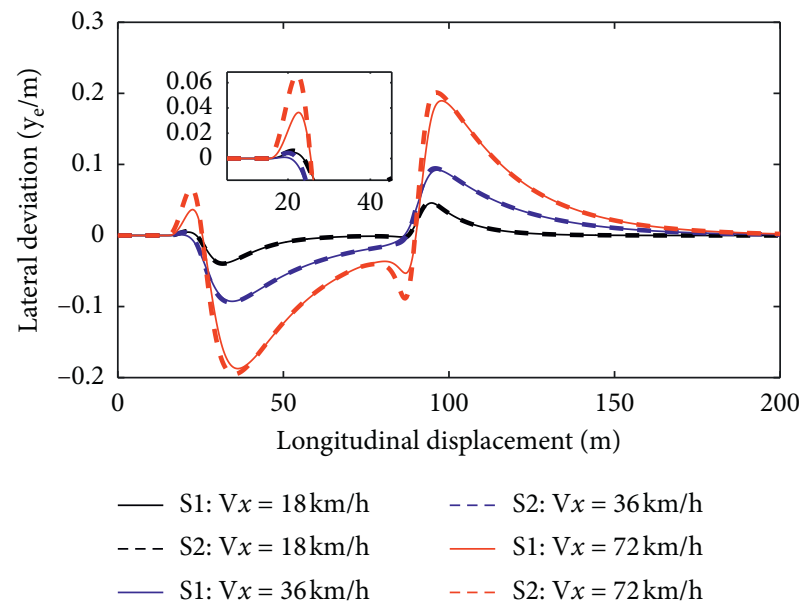

(b)

FIgURE 9: The effect comparison between S1 and S2 under different velocity conditions. (a) Orientation deviation. (b) Lateral deviation.
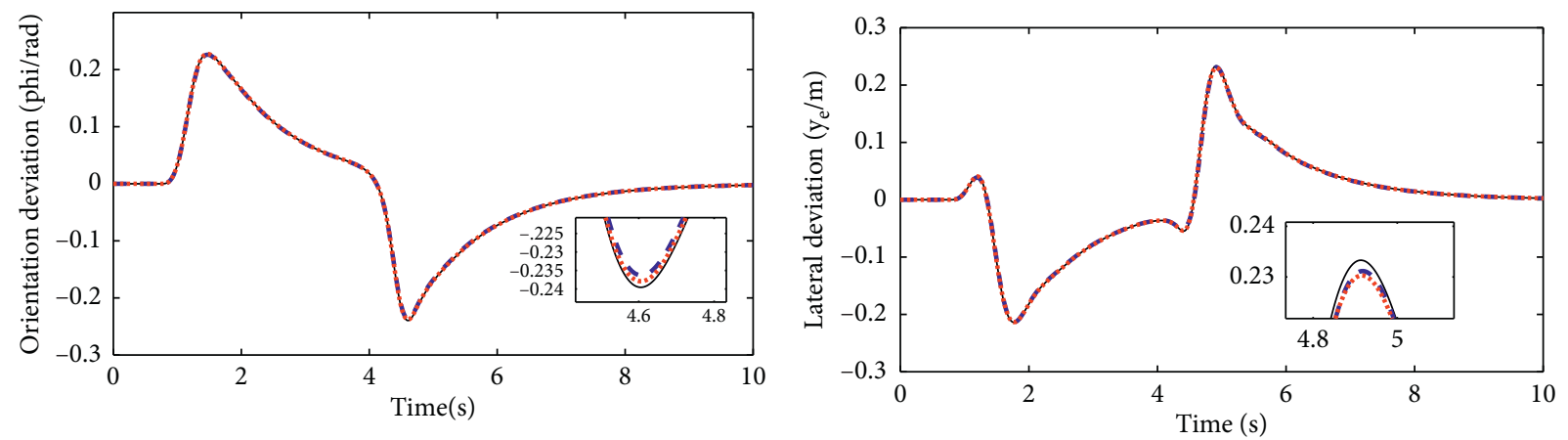

$-\mathrm{B} 1$
$---\mathrm{B} 2$
$\ldots .$.
$\mathrm{B} 3$

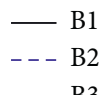

(a)

(b)

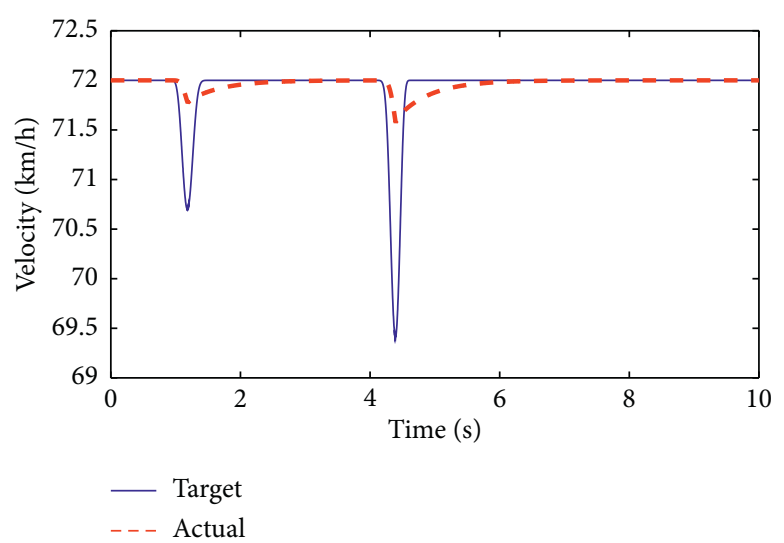

(c)

Figure 10: The effect comparison between B1, B2, and B3 under $36 \mathrm{~km} / \mathrm{h}$. (a) Orientation deviation. (b) Lateral deviation. (c) Velocity.

\section{Hardware-in-the-Loop (HIL) Test}

In this section, hybrid control unit HIL tests are adopted to verify the correctness of the simulation results in Section 4 . The HIL test bench is shown in Figure 14.
The test conditions are assumed to be the same as those in Section 4.1. The comparison results for the IV path-tracking system without subsystems are shown in Figure 15, and the results for the IV path-tracking system with subsystems are in Figure 16. 

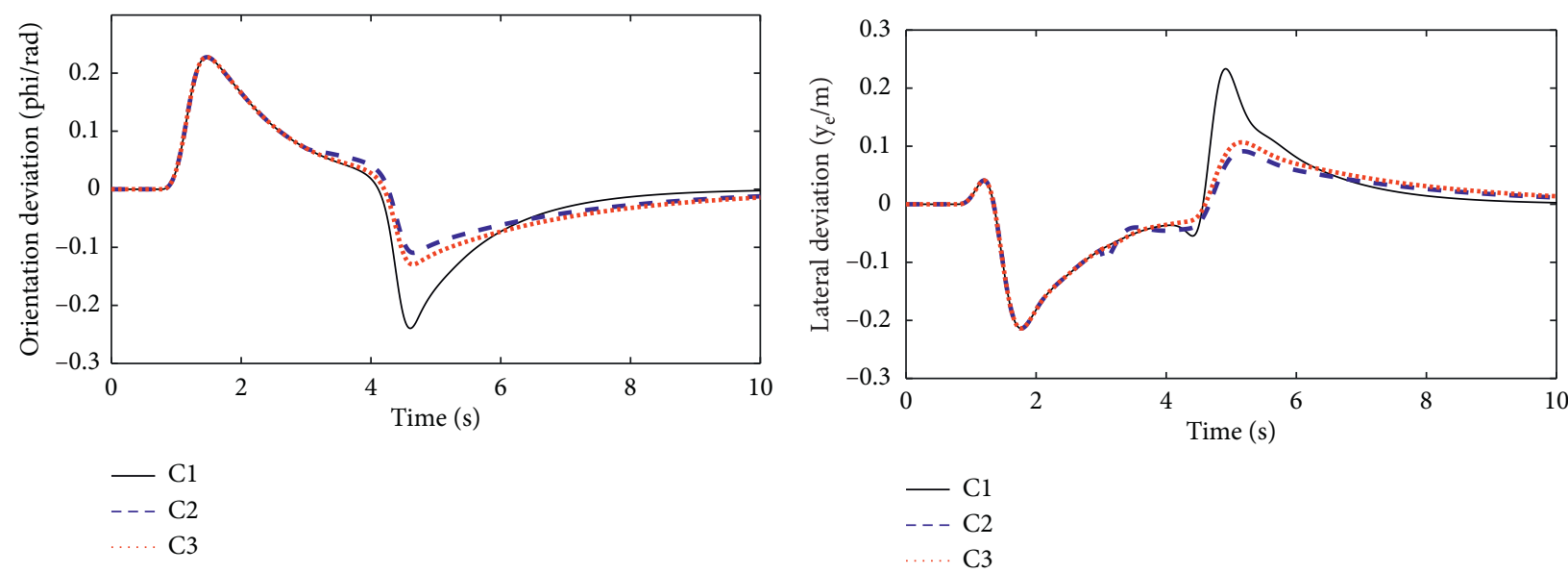

(a)

(b)

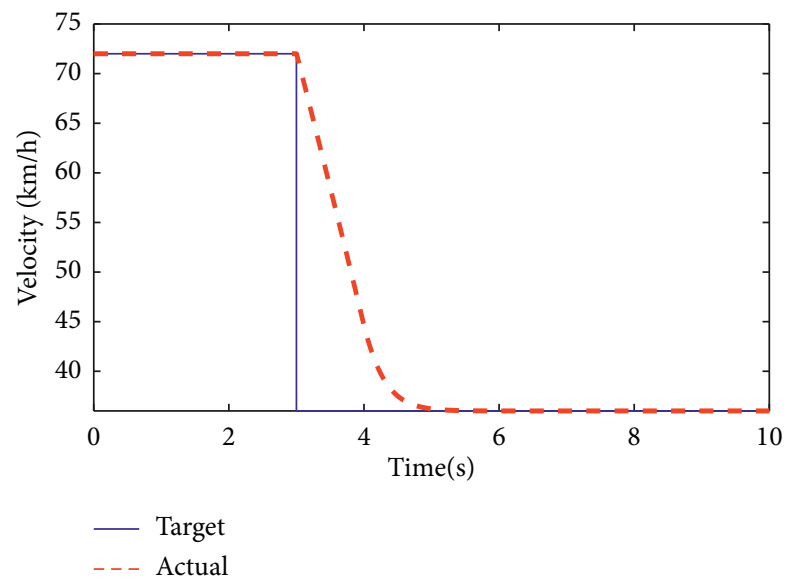

(c)

Figure 11: The effect comparison between C1, C2, and C3 under given velocity conditions. (a) Orientation deviation. (b) Lateral deviation. (c) Velocity.

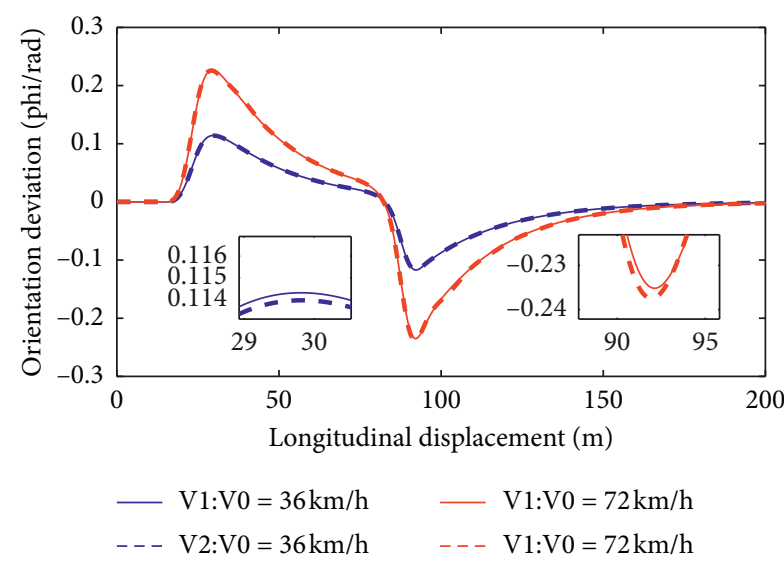

(a)

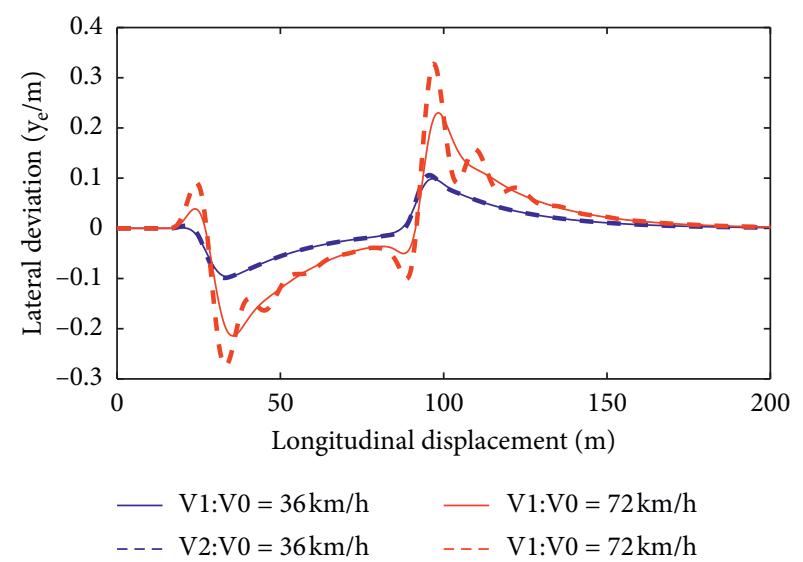

(b)

Figure 12: The effect comparison between V1 and V2 different velocity conditions. (a) Orientation deviation. (b) Lateral deviation.

Figures 15 and 16 depict that the curves of orientation and lateral deviations for the HIL and simulation results are nearly the same without fluctuation when the velocity is $36 \mathrm{~km} / \mathrm{h}$. That is, the designed IV path-tracking control system can operate normally on the HIL platform. The peak deviation values of the simulation and HIL test results in Figure 15 are 0.098 and $0.106 \mathrm{~m}$, respectively. The corresponding values in Figure 16 are 0.106 and 


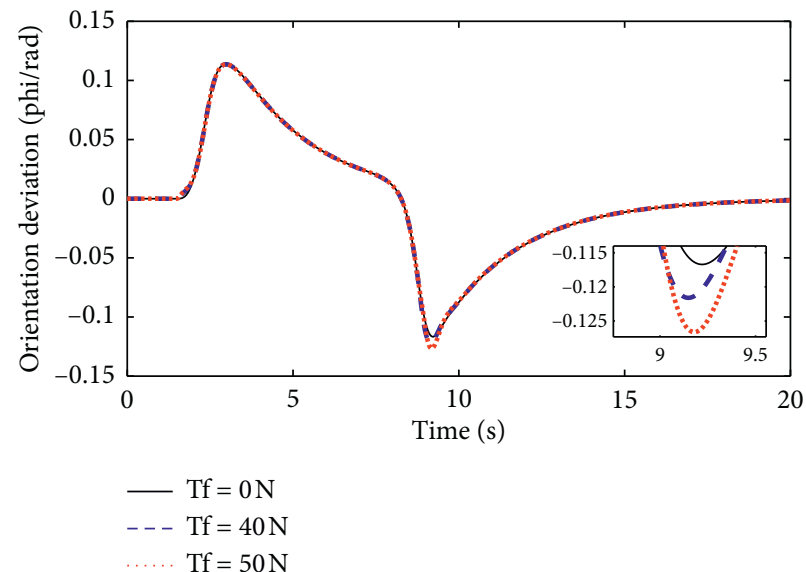

(a)

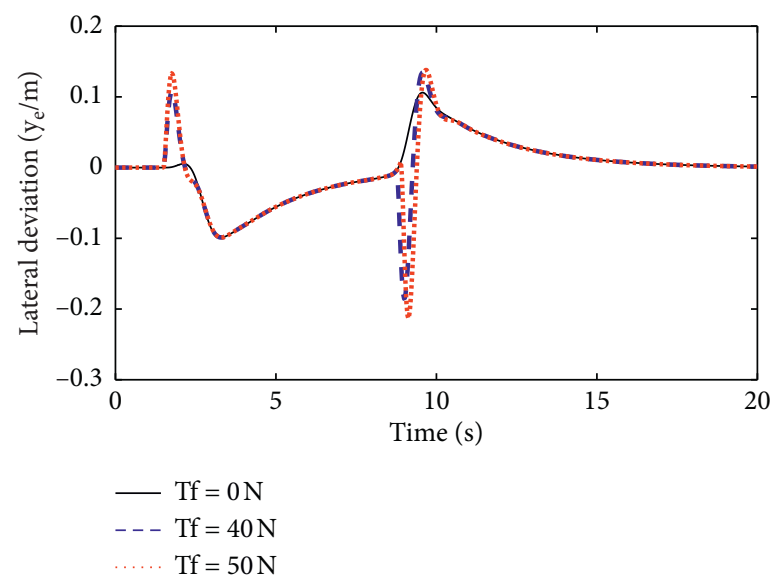

(b)

FIGURE 13: Influence of different interference torque on the path-tracking stability of the intelligent vehicle. (a) Orientation deviation. (b) Lateral deviation.

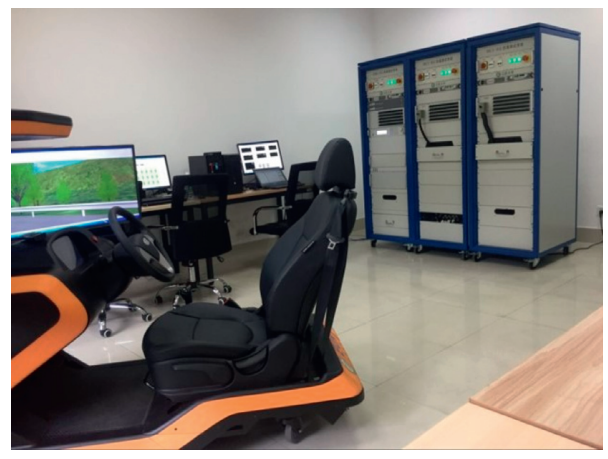

FIgURe 14: HCU HIL platform.
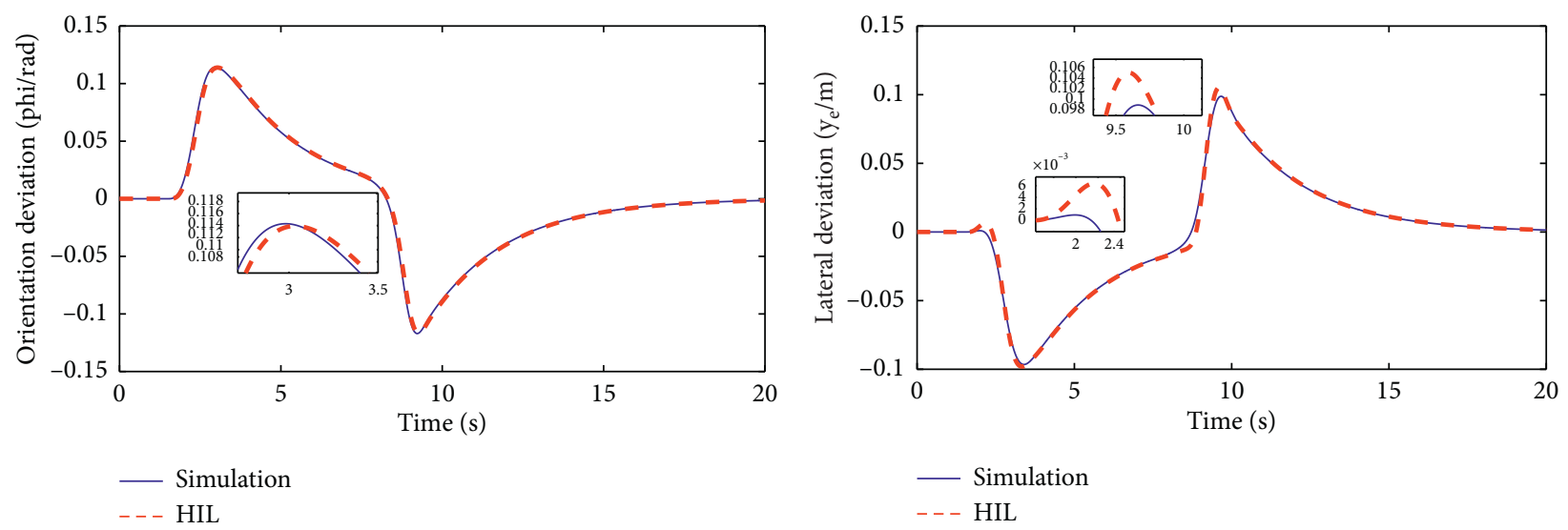

(a)

(b)

FIGURE 15: The effect comparison of the IV path tracking system without subsystems. (a) Orientation deviation. (b) Lateral deviation. 


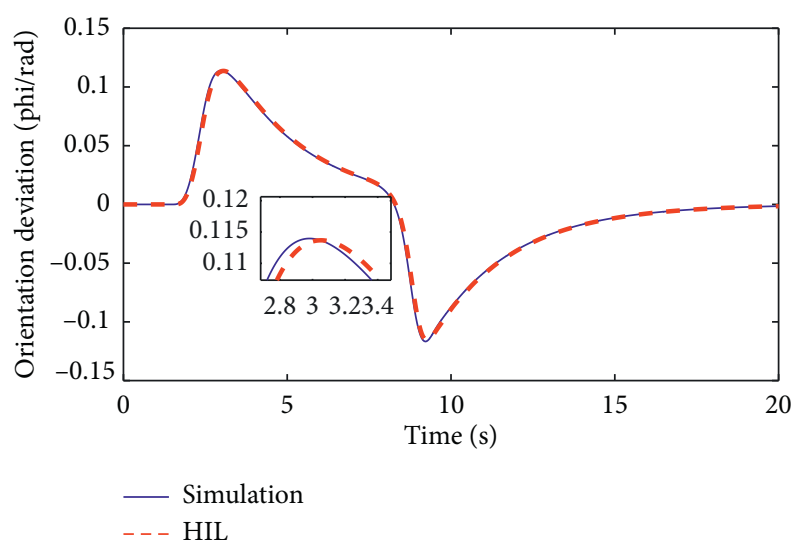

(a)

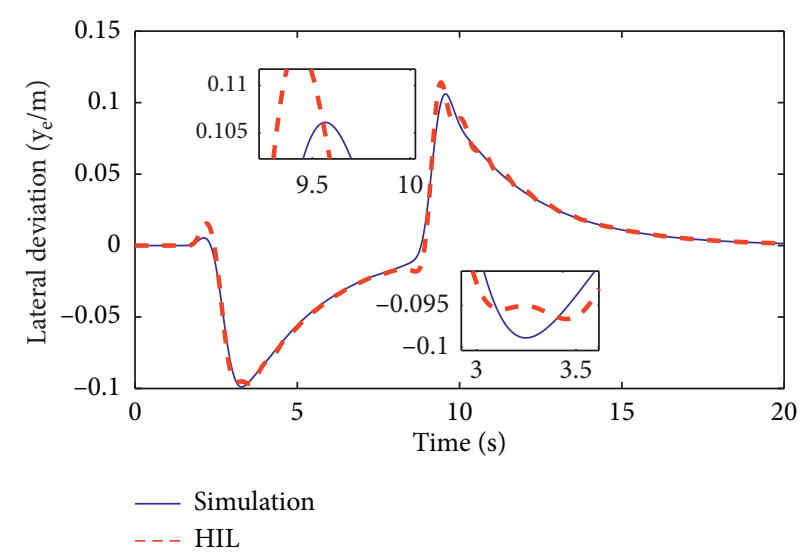

(b)

FIGURE 16: The effect comparison of the IV path tracking system with subsystems. (a) Orientation deviation. (b) Lateral deviation.

$0.114 \mathrm{~m}$, which imply that the subsystems have a certain influence on the accuracy of the IV path-tracking system. The curves of the IV path-tracking system from the HIL test are slightly offset from those of the simulation results due to the system delay caused by controller transcoding, signal $\mathrm{I} / \mathrm{O}$ and $\mathrm{O} / \mathrm{I}$ conversion, and $\mathrm{O} / \mathrm{I}$ conversion. The designed controller meets the real-time requirements.

\section{Conclusion}

This paper presents an MIHC algorithm with subsystems to reduce the performance conflict between a path-tracking motion system and its subsystems during the IV motion control process. The working principle of MIHC and the corresponding dynamic models are introduced first. Then, controllers at different levels are proposed on the basis of MIHC. Lastly, the simulations and HIL test of the IV pathtracking system with or without subsystems are carried out under different conditions. The obtained results imply that ASS has a great influence on the accuracy of the IV trajectory-tracking system compared with ABS, especially in the lateral deviation of the IV trajectory-tracking system. ABS has a considerable influence on the tracking stability of the IV trajectory-tracking system. Further study on the experimental implementation of the controller through IVs will be conducted in the near future.

\section{Data Availability}

The raw/processed data required to reproduce these findings cannot be shared at this time as the data also form part of an ongoing study.

\section{Conflicts of Interest}

The authors declare that they have no conflicts of interest.

\section{Acknowledgments}

This work was supported by the Program of the Youth Natural Science Foundation of Jiangsu Province (Grant no.
BK20200909) and the Program of the China Postdoctoral Science Foundation (Grant no. 2020M671381).

\section{References}

[1] Q. Ye, R. Wang, Y. Cai, X. Xu, X. Meng, and C. Long, "A study of the novel vision guided IV trajectory tracking control system based on expected yaw velocity," Advances in Engineering Software, vol. 131, pp. 196-204, 2019.

[2] Y. Wang, Y. F. Cai, and L. Chen, "Design of intelligent and connected vehicle path tracking controller based on model predictive control," Journal of Mechanical Engineering, vol. 55, no. 8, pp. 136-144, 2019.

[3] J.-B. Receveur, S. Victor, and P. Melchior, "Robust longitudinal motion planning using vehicle model inversion," IFACPapersOnLine, vol. 52, no. 5, pp. 103-108, 2019.

[4] H. Zhou, F. Jia, H. Jing, Z. Liu, and L. Guvenc, "Coordinated longitudinal and lateral motion control for four wheel independent motor-drive electric vehicle," IEEE Transactions on Vehicular Technology, vol. 67, no. 5, pp. 3782-3790, 2018.

[5] F. Lin, Y. Zhang, Y. Zhao et al., "Trajectory tracking of autonomous vehicle with the fusion of DYC and longitudinal-lateral control," Chinese Journal of Mechanical Engineering, vol. 32, pp. 1-16, 2019.

[6] T. Chen, X. Xu, L. Chen, H. Jiang, Y. Cai, and Y. Li, "Estimation of longitudinal force, lateral vehicle speed and yaw rate for four-wheel independent driven electric vehicles," Mechanical Systems and Signal Processing, vol. 101, pp. 377388, 2018.

[7] Y. Huang, H. Wang, A. Khajepour, H. Ding, K. Yuan, and Y. Qin, "A novel local motion planning framework for autonomous vehicles based on resistance network and model predictive control," IEEE Transactions on Vehicular Technology, vol. 69, no. 1, pp. 55-66, 2020.

[8] C. Hu, Z. Wang, and H. Taghavifar, "MME-EKF-based pathtracking control of autonomous vehicles considering input saturation," IEEE Transactions on Vehicular Technology, vol. 68, no. 6, pp. 1-11, 2019.

[9] L. Tang, J. Huang, L.-M. Zhu, X. Zhu, and G. Gu, "Path tracking of a cable-driven snake robot with a two-level motion planning method," IEEE/ASME Transactions on Mechatronics, vol. 24, no. 3, pp. 935-946, 2019.

[10] P. Hang, X. Chen, and F. Luo, "LPV/Ho controller design for path tracking of autonomous ground vehicles through four- 
wheel steering and direct yaw-moment control," International Journal of Automotive Technology, vol. 20, no. 4, pp. 679-691, 2019.

[11] M. Samuel, M. Maziah, M. Hussien, and N. Y. Godi, "Control of autonomous vehicle using path tracking: a review," $A d$ vanced Science Letters, vol. 24, no. 6, pp. 3877-3879, 2018.

[12] L. Zhang and G. Wu, "Combination of front steering and differential braking control for the path tracking of autonomous vehicle," SAE Technical Paper, vol. 1, no. 1627, pp. 1-12, 2016.

[13] R. Liu, M. Wei, N. Sang, and J. Wei, "Research on curved path tracking control for four-wheel steering vehicle considering road adhesion coefficient," Mathematical Problems in Engineering, vol. 2020, Article ID 3108589, 18 pages, 2020.

[14] X. Ji, X. He, C. Lv, Y. Liu, and J. Wu, "Adaptive-neuralnetwork-based robust lateral motion control for autonomous vehicle at driving limits," Control Engineering Practice, vol. 76, pp. 41-53, 2018.

[15] R. Wang, Q. Ye, Y. Cai et al., "Analyzing the influence of automatic steering system on the trajectory tracking accuracy of intelligent vehicle," Advances in Engineering Software, vol. 121, pp. 188-196, 2018.

[16] H. Wang, B. Liu, X. Ping, and Q. An, "Path tracking control for autonomous vehicles based on an improved MPC," IEEE Access, vol. 99, pp. 1-9, 2019.

[17] J. Yu, X. Guo, X. Pei et al., "Path tracking control based on tube MPC and time delay motion prediction," IET Intelligent Transport Systems, vol. 14, no. 6, pp. 1-12, 2019.

[18] H. Du and N. Zhang, "Robust stability control of vehicle rollover subject to actuator time delay," Proceedings of the Institution of Mechanical Engineers, Part I: Journal of Systems and Control Engineering, vol. 222, no. 3, pp. 163-174, 2008.

[19] G. Huang, W. Wang, W. Chen, and L. He, "Single-point preview driver and non-linear tyre model-based direct yaw moment control method for vehicles," in Proceedings of the 36th Chinese Control Conference, pp. 9419-9424, Dalian, China, July 2017.

[20] D. Lee, K. S. Kim, and S. Kim, "Controller design of an electric power steering system," IEEE Transactions on Control Systems Technology, vol. 99, pp. 1-8, 2018.

[21] S. B. Choi, "Antilock brake system with a continuous wheel slip control to maximize the braking performance and the ride quality," IEEE Transactions on Control Systems Technology, vol. 16, no. 5, pp. 996-1003, 2008.

[22] J. Emmerson, "Speeds of cars on sharp horizontal curves," Traffic Engineering \& Control, vol. 11, no. 3, pp. 135-137, 1969.

[23] E. H. Karam and N. Mjeed, "Adaptive backstepping SMC with cuckoo search algorithm for two wheeled self balancing robot," International Journal of Engineering \& Technology, vol. 7, no. 3.13 , pp. $27-32,2018$. 